\title{
IMPACT OF WATER DROPS ON THE VISIBLE RADIATION TRANSMITTANCE OF GLAZINGS UNDER OUTSIDE RADIANT CONDITIONS
}

\author{
I. V. POLLET, J. G. PIETERS ${ }^{\dagger}$ and R. VERSCHOORE \\ Biosystems Engineering, Ghent University, Coupure Links 653, 9000 Ghent, Belgium
}

Accepted 21 October 2002

\begin{abstract}
Experimentally determined directional-hemispherical transmittances for visible radiation were used to calculate the effect of condensate on the hemispherical-hemispherical transmittance values under diffuse radiant conditions for single glass and polyethylene. The hemispherical-hemispherical transmittances were determined in the horizontal plane for different inclinations of both glazing materials assuming a completely overcast sky and a clear sky. In all cases, condensate was found to reduce the transmittance. Under an overcast sky, the transmittance of single glass was reduced by the presence of condensate from 8 up to $13 \%$ (on a relative scale) for increasing inclination angles of the glass plate, while for the polyethylene film, the transmittance reduction due to condensate decreased from 20 down to $13 \%$. Under a clear sky, depending on date, time, and orientation of the glazing material, the transmittances of single glass and polyethylene were reduced by between 6 and $15 \%$ and by between 8 and $21 \%$, respectively, due to the presence of condensate. These results revealed that the impact of the presence of condensate on the transmittance of a glazing cannot be omitted from design and performance calculations of solar energy systems.

(c) 2003 Elsevier Science Ltd. All rights reserved.
\end{abstract}

\section{INTRODUCTION}

During the last decade, due to the need for more reliable energy studies of buildings and solar energy systems, an increasing interest in the scientific world in modelling and measuring the angular dependent transmittance of transparent materials has arisen (Platzer, 1992; Hutchins and Ageorges, 1993; Gombert et al., 1995; Palmer et al., 1996; Mbise et al., 1997; Roos, 1997; Van Nijnatten and Spee, 1997; Rubin et al., 1998, 1999; Karlsson and Roos, 2000a,b; Karlsson et al., 2001). Based on these angular transmittances, the hemispherical transmittances under diffuse outside radiant conditions can be calculated. Besides, transparent materials are often poorly insulating, thin, transmitting materials, such as single glass or plastic films, on which condensation frequently occurs on the non-irradiated surface of the transparent material when the inside relative humidity is high. These conditions which give rise to the occurrence of condensation, are met in solar stills (El-Bahi and Inan, 1999; Aggarwal and Narayan, 2000) and occur frequently in greenhouses (von Zabeltitz, 1987; Briscoe

\footnotetext{
${ }^{\dagger}$ Author to whom correspondence should be addressed. Tel. +329-264-61-88; fax: +329-264-62-35; e-mail: Jan.Pieters@rug.ac.be
}

and Galvin, 1991; Geoola et al., 1994, 1998; Schultz and Bartnig, 1996; Pieters and Deltour, 1998) and even in flat plate solar collectors (Hsieh and Rajvanshi, 1977; Fechner and Bucek, 1999).

Against this background, the directional-hemispherical transmittances $\left(\tau_{\mathrm{dh}}\right)$ of a clear single glass (SG) plate and a low-density polyethylene (LDPE) film were measured in the laboratory by Pollet and Pieters (2002b) as a function of incidence angle and wavelength in the visible radiation range. The laboratory unit was the one described in full detail in Pollet and Pieters (1999). It consisted of a hot box and a cold box, separated by an insulated wall in which the SG plate or the LDPE film under investigation were placed. Increasing the humidity in the hot box by means of an accurate humidity control system and lowering the temperature in the cold box to a few degrees above freezing point allowed condensation (without run-off) to be generated on the test material. In the cold box a halogen lamp was used as the collimated light source. Use of spectral filters allowed radiation beams with wavelengths of $400,450,500,550,600,650$, and $700 \mathrm{~nm}$ to be produced. The beam was directed at the prechosen incidence angle on the test material, where it was partly transmitted into the hot box. In the hot box, a large integrating sphere captured the transmitted radiation, while its flux was deter- 
mined using a photodetector mounted on the sphere.

The angular transmittance curves of the SG plate and the LDPE film were determined once in the dry state and once when covered with condensate and are given in Fig. 1. The curves for the dry state show the typical trend of a dry transparent and uniform material: at normal incidence the transmittance is maximal and it remains nearly constant for small incidence angles. For higher angles of incidence, an increasing transmittance reduction for incidence angles of up to $90^{\circ}$ occurs. As can be deduced from Fig. 1, the plateau of the transmittance curve is somewhat longer for SG than for LDPE.

Furthermore, Fig. 1 illustrates that the impact of the condensate on the transmittance is angle dependent: no effect up to a considerable transmittance reduction is observed. For small angles of incidence, no transmittance difference between the dry and the wet state of SG is found, while a relative transmittance reduction of $25 \%$ is observed on LDPE due to the presence of condensate. At higher incidence angles, condensate gives rise to a transmittance decrease on SG, while the transmittance reduction on LDPE diminishes with increasing incidence angle. As shown by Pieters et al. (1997) via simulations, the results can be explained by the fact that flat condensate drops ('pancakes') are formed on the glass plate, whereas the drops on the plastic film are more curved and approach a hemispherical shape. The kind of condensate pattern formed depends on the surface tension of the material. Furthermore, it was shown by Pollet and Pieters (2002a) that the directionalhemispherical light transmittance of a transparent material covered with condensate does not significantly change for different inclination angles

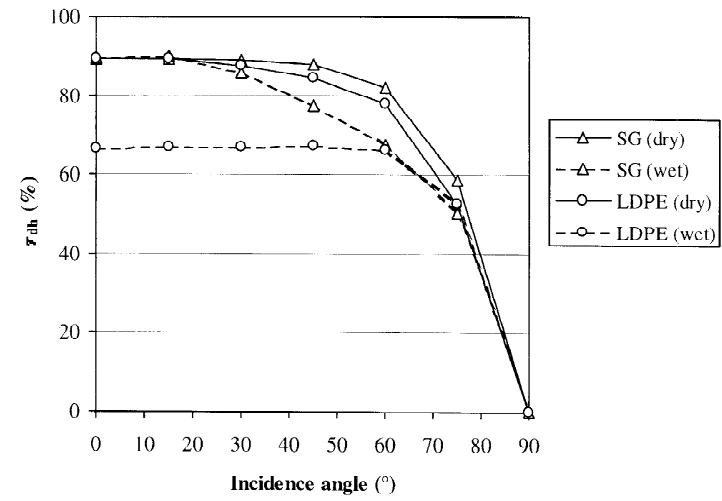

Fig. 1. Directional-hemispherical transmittance for visible radiation of (SG) single glass and (LDPE) low-density polyethylene in the dry state and when covered with condensate. of the material. This implies that independently of the inclination of the wet glazing, the same directional-hemispherical transmittance values can be applied to determine the hemispherical-hemispherical transmittance values of any inclined wet glazing.

In practice, even under a cloudless sky, solar radiation strikes the window of a house or an office building or the covering material of a nontracking solar collector at a large range of incidence angles, since at least part of the total solar radiation is diffused due to atmospheric scattering. In that case, the hemispherical-hemispherical transmittance $\left(\tau_{\mathrm{hh}}\right)$ can be an appropriate measure for the transmittance of a glazing which can be derived from the directional-hemispherical transmittance curves.

In this study, the hemispherical-hemispherical transmittance values of a SG sheet $(4 \mathrm{~mm})$ and an LDPE film $(150 \mu \mathrm{m})$ were calculated for different inclinations and orientations assuming a completely overcast sky and a fully clear sky, in order to analyse to which extent the presence of condensate affects the total transmittance of transparent materials under different outside solar radiation conditions.

\section{CALCUlATION METHODS AND CIRCUMSTANCES}

\subsection{Model structure}

2.1.1. General description. A numerical model was developed to calculate the fluxes of direct and diffuse radiation incident on and transmitted through a transparent material under a fully overcast sky and under a clear sky. In that way, the hemispherical-hemispherical transmittance values could be obtained. Radiation was assumed to be unpolarised and to strike the transparent material at one side. Calculations could be performed for any orientation of the transparent material (determined by the azimuthal angle and the inclination angle) and for any particular moment of the year. The transmittances were determined for radiation measured in the horizontal plane.

The nomenclature used for the calculations is illustrated in Fig. 2 for the case of an east oriented glazing. The position of the sun is defined by the azimuthal angle $\phi_{\text {sun }}$ and the altitude angle $\theta_{\text {sun }}$ of the sun, whereas any point $\mathrm{P}$ of the sky is defined by its azimuthal angle $\phi$ and altitude angle $\theta$ (Rea, 1993). The zenith angle $\zeta$ between the zenith $\mathrm{Z}$ and point $\mathrm{P}$ is the complementary angle 


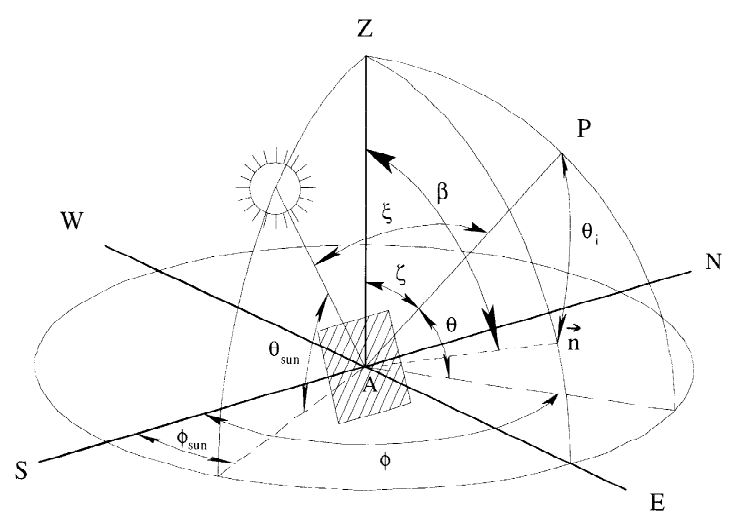

Fig. 2. Scheme of solar radiation striking an east oriented glazing with the symbols used.

of the altitude angle $\theta$. The angle between the sun and the sky point is given by $\xi$. The inclination angle of the transparent material is equal to $\beta$, whereas $\vec{n}$ represents the normal to the glazing material. Furthermore, the incidence angle of a ray departing from the sky point $\mathrm{P}$ and striking the transparent material in point $\mathrm{A}$ is given by $\theta_{\mathrm{i}}$.

The hemispherical-hemispherical transmittance $\tau_{\mathrm{hh}}(\beta)$ of a transparent material at an inclination angle $\beta$ was calculated as the ratio of the irradiances $E_{\mathrm{t}}$ and $E_{\mathrm{i}}$ of the transmitted and the incident beam, respectively. As a consequence, the hemispherical-hemispherical transmittance $\tau_{\mathrm{hh}}(\beta)$ for radiation measured in the horizontal plane can be deduced from the directional-hemispherical transmittance and the sky radiance distribution as follows:

$$
\begin{aligned}
& \int_{\mathrm{hh}}(\beta)=\frac{E_{\mathrm{t}}}{E_{\mathrm{i}}}= \\
& \frac{\int_{0}^{2 \pi / 2} \int_{0}^{\pi / 2} \tau_{\mathrm{dh}}\left(\theta_{\mathrm{i}}\right) L(\theta, \phi) \cos \varsigma \cos \theta \mathrm{d} \theta \mathrm{d} \phi}{\int_{0}^{2 \pi} \int_{0}^{\pi / 2} L(\theta, \phi) \cos \cos \theta \mathrm{d} \theta \mathrm{d} \phi} \\
& \text { for }\left|\theta_{\mathrm{i}}\right| \leq \pi / 2
\end{aligned}
$$

where $\tau_{\mathrm{dh}}\left(\theta_{\mathrm{i}}\right)$ is the directional-hemispherical transmittance for radiation incident at an angle $\theta_{\mathrm{i}}$, $L(\theta, \phi)\left(\mathrm{W} \mathrm{sr}^{-1} \mathrm{~m}^{-2}\right)$ is the sky radiance, $\beta(\mathrm{rad})$ is the inclination angle of the transparent material, $\theta, \phi$, and $\theta_{\mathrm{i}}$ represent the altitude angle, the azimuthal angle, and the incidence angle of the sky point under consideration, respectively.

It is clear from Eq. (1) that directional-hemispherical transmittance values were used as input data to calculate the hemispherical-hemispherical transmittance values. The inclination angle $\beta$ of the transparent material was taken into account via the incidence angle $\theta_{\mathrm{i}}$ of the radiation. Under a clear sky, the hemispherical-hemispherical transmittance depends also on the azimuthal angle of the surface. This azimuthal angle was expressed with respect to the south.

Under a clear sky, the hemispherical-hemispherical transmittance changes over time. In that case, a time and irradiance weighted hemispherical-hemispherical transmittance value has to be determined as a measure for the hemisphericalhemispherical transmittance. The weighted average hemispherical-hemispherical transmittance $\bar{\tau}_{\mathrm{hh}}(\beta)$ equals the ratio of the total amount of transmitted and incident radiation. This means that the instantaneous transmittance values had to be weighted according to the actual outside irradiance level before integration. The weighted average hemispherical-hemispherical transmittance $\bar{\tau}_{\mathrm{hh}}(\beta)$ in the time interval between the moments $t_{1}$ and $t_{2}$ is given by:

$$
\bar{\tau}_{\mathrm{hh}}(\beta)=\frac{\int_{\mathrm{t}_{1}}^{\mathrm{t}_{2}} \tau_{\mathrm{hh}}(\beta) E_{\mathrm{i}} \mathrm{d} t}{\int_{\mathrm{t}_{1}}^{\mathrm{t}_{2}} E_{\mathrm{i}} \mathrm{d} t}
$$

2.1.2. Sky radiance distribution. According to Eq. (1), the transmittance depends on the radiance distribution of the sky. Under an overcast sky, no direct radiation is available and the radiance distribution of the sky is supposed to be independent of the solar position. This means that the hemispherical-hemispherical transmittance of the transparent material under an overcast sky did not depend on date and time and azimuthal orientation of the transparent material. In this study, the Moon and Spencer distribution was used, since this distribution is also adopted by the Commission Internationale de l'Eclairage (CIE) (Rea, 1993). According to this distribution, the zenith radiance is three times higher than at the horizon:

$L=\frac{L_{\mathrm{z}}}{3}(1+2 \sin \theta)$

where $L_{\mathrm{z}}\left(\mathrm{W} \mathrm{sr}^{-1} \mathrm{~m}^{-2}\right)$ is the sky zenith radiance.

In the case of a clear sky, the sky radiance as well as its distribution depend on the relative position of the sun. The solar position is specified by the solar altitude and solar azimuth, and is a function of site latitude, solar time, and solar declination. 
The standard clear sky radiance distribution function for diffuse radiation developed by Kittler (1967) and adopted by the CIE, was used. This function is given by:

$L=L_{\mathrm{z}}$

$\frac{\left(0.91+10 \mathrm{e}^{-3 \xi}+0.45 \cos ^{2} \xi\right)\left(1-\mathrm{e}^{-0.32 / \cos \mathrm{s}}\right)}{\left(0.91+10 \mathrm{e}^{-3 \theta_{\text {sun }}}+0.45 \sin ^{2} \theta_{\text {sun }}\right)\left(1-\mathrm{e}^{-0.32}\right)}$

According to this equation, the radiance of the diffuse radiation is highest in the sky vault element surrounding the sun. The zenith radiance $L_{\mathrm{z}}$, which is a function of the solar altitude and the total horizontal irradiance coming from the sky, was taken from Rea (1993).

The irradiance of the direct radiation was estimated from the total diffuse irradiance, taking into account the ratio of diffuse and direct radiation. The distribution of the total solar radiation over diffuse and direct radiation changes with the altitude of the sun (Monteith and Unsworth, 1990). The fraction of the diffuse radiation decreases with higher solar altitude. These values are given in Table 1 and were incorporated into the model.

The visible radiation fractions of direct and diffuse radiation are different from each other and depend also on solar altitude. The ratio of visible radiation to total radiation increases with increasing altitude angle for direct and diffuse radiation. The radiometric visible radiation fractions for direct and diffuse radiation as given by Monteith (1973) were taken into account (see Table 1). Differences in the spectral distribution between direct and diffuse radiation were not taken into account since uniform data were not available. However, since the transmittance values of SG and LDPE are nearly constant over the visible radiation range, errors introduced in this way were very small.

\subsection{Simulation circumstances}

The directional-hemispherical transmittance curves in the visible radiation range, as presented in Fig. 1, were used as input data in Eq. (1) to calculate the hemispherical-hemispherical transmittances. The transmittances for visible radiation were derived from spectral measurements at an interval of $50 \mathrm{~nm}$ in the wavelength range from 400 to $700 \mathrm{~nm}$, taking into account the ISO 9845 1 standard for direct normal irradiance of a sky with an air mass of 1.5. All calculations were performed for the dry state and the condensate covered state.

Under an overcast sky, the hemisphericalhemispherical transmittances in the horizontal plane of SG and LDPE were calculated for inclination angles between 0 and $90^{\circ}$ at $10^{\circ}$ intervals. Under clear sky conditions, the average hemispherical-hemispherical transmittances in the horizontal plane of SG and LDPE were calculated. Ghent with a latitude of $51^{\circ}$ and a longitude of $-3.5^{\circ}$ was chosen as the location. The calculations were performed for every half an hour of three characteristic days: the summer (22 June) and the winter (23 December) solstice and the spring equinox (22 March). The radiance distribution on the autumn equinox (22 September) is similar to that on the spring equinox. The time weighted transmittance was calculated according to Eq. (2). Furthermore, a north (N), an east (E), and a south (S) oriented transparent material were considered. A west (W) oriented material shows analogous transmittance characteristics as an E-oriented one. Therefore, it was not treated separately. The inclination of the transparent material was set at $0,30,60$, and $90^{\circ}$.

\section{RESULTS AND DISCUSSION}

\subsection{Overcast sky}

3.1.1. Dry state. The hemispherical-hemispherical transmittances of dry and wet SG and LDPE under an overcast sky are given in Fig. 3 as a function of the inclination angle of the glazing. The relative effect of the condensate on the transmittance of both glazing materials is also illustrated in Fig. 3. For both glazing materials, the hemispherical-hemispherical transmittance decreased gradually with increasing inclination angle. It should be stressed that the transmittance

Table 1. Fraction of diffuse radiation in total radiation and fraction of visible radiation in direct and diffuse radiation as a function of solar altitude (Monteith, 1973; Monteith and Unsworth, 1990)

\begin{tabular}{lllllllll}
\hline Fraction & \multicolumn{7}{l}{ Solar altitude $\left[{ }^{\circ}\right]$} \\
\cline { 2 - 9 } & 5 & 10 & 20 & 30 & 40 & 50 & 60 & 70 \\
\hline Diffuse radiation in total radiation & 0.76 & 0.60 & 0.47 & 0.36 & 0.29 & 0.23 & 0.21 & 0.20 \\
Visible radiation in direct radiation & 0.20 & 0.28 & 0.37 & 0.40 & 0.42 & 0.43 & 0.43 & 0.43 \\
Visible radiation in diffuse radiation & 0.61 & 0.62 & 0.63 & 0.65 & 0.67 & 0.70 & 0.73 & 0.76
\end{tabular}




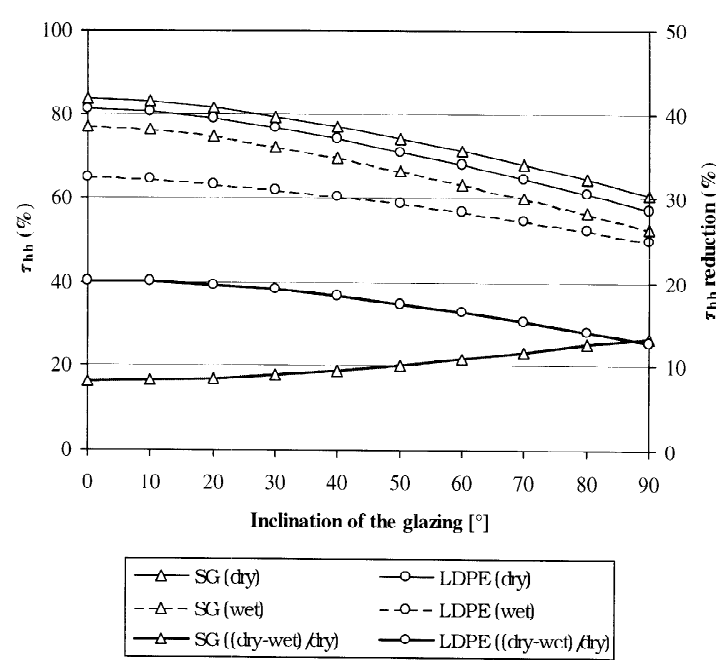

Fig. 3. Hemispherical-hemispherical transmittances of dry and wet SG and LDPE and the relative transmittance difference between the dry and the wet state for an overcast sky as a function of the inclination angle of the glazing material.

is not a measure for the amounts of radiation found under or behind the glazing. Apart from the transmittance, the amount of incident radiation is of importance. The latter can vary a lot depending on time, inclination and orientation of the material.

The transmittance of dry SG determined in the horizontal plane, was found to decrease from 84 to $61 \%$ with increasing inclination angle. The highest radiant intensity occurs in the zenith, which on a horizontal surface corresponded with a zero incidence angle for which the directional transmittance - as shown in Fig. 1-is maximal. In the case of a vertical surface, the lowest radiant intensities are found at small incidence angles. This gave rise to an obviously greater hemispherical-hemispherical transmittance for a horizontal surface than for a vertical surface under an overcast sky. As a result, the directional-hemispherical transmittance decreased with increasing incidence angle.

Edwards and Lake (1964, 1965) measured solar transmittances of glass plates under overcast sky conditions ranging from 77 to $83 \%$ for a roof slope of $26^{\circ}$. The value of $80 \%$, simulated in this study, is in the middle of this range. A hemispherical-hemispherical transmittance of $83 \%$ was measured by Breuer (1981) on horizontal glass under an overcast sky. This value is very similar to the transmittance of $83.7 \%$ predicted in this study.

When considering the LDPE film in the dry state, the hemispherical-hemispherical transmittance of the plastic film decreased gradually from
$81 \%$ for a horizontal surface to $57 \%$ for a vertical surface (see Fig. 3).

As can be observed in Fig. 3, the hemispherical-hemispherical transmittance curves of SG and LDPE in the dry state were parallel, since the directional-hemispherical transmittance curves of both materials were almost similar. The highest transmittance-and consequently the irradiance level-under the glazing was found for SG. The hemispherical-hemispherical transmittances of the LDPE film were on average 3\% lower than the SG transmittances. The average relative difference was $4 \%$.

3.1.2. Wet state. For both materials, condensate reduced the total transmittance under overcast sky conditions, as can be observed in Fig. 3. However, due to the different condensate patterns formed on both glazings, the influence of the condensate on the transmittance differed obviously for both materials. Moreover, the inclination angle of the glazing material played an important role in the effect of the condensate on the total transmittance. The angular transmittance values of a wet glazing, however, do not change as a function of the inclination, as shown by Pollet and Pieters (2002a).

The hemispherical-hemispherical transmittance of wet SG was found to be a curve nearly parallel to the one of dry SG. The absolute transmittance difference between the dry and the wet state was on average $7 \%$. Relatively, the effect of condensate on the hemispherical-hemispherical transmittance increased with increasing inclination angle, as shown in Fig. 3. As was shown in Fig. 1, the directional-hemispherical transmittance of SG was most affected by the presence of condensate at high angles of incidence. Since the fraction of high incidence angle radiation increased with increasing inclination angle, the effect of condensate on the hemispherical-hemispherical transmittance also increased with increasing inclination angle of the SG plate. The decrease of the hemispherical-hemispherical transmittance due to condensate varied from $8 \%$ for a horizontal surface up to $13 \%$ for a vertical one.

It can be concluded that under a glasshouse roof with a slope of 20 to $30^{\circ}$, the irradiance is reduced by about $9 \%$, when the roof is covered with condensate under an overcast sky. These findings fully agree with those of von Zabeltitz (1987), measured by means of an experimental set-up. For 15 and $30^{\circ}$ inclined SG plates, this author found a relative reduction of $9 \%$ for both inclination angles. For vertical glass surfaces as 
found in houses or office buildings, the transmittance was even more reduced by the condensate.

When covered with condensate, the relative reduction of the transmittance of LDPE due to the presence of condensate varied from $20 \%$ for a horizontal film down to $13 \%$ for a vertical film. The hemispherical-hemispherical transmittance of a horizontal LDPE film was reduced from $81 \%$ to a value of $65 \%$. For a vertical wet LDPE film, $50 \%$ of the incident radiation was transmitted, instead of 57\% for dry LDPE. The lower impact of the condensate on the hemispherical-hemispherical transmittance for increasing inclination angles of the film can be explained via the directional-hemispherical transmittance of LDPE. As shown in Fig. 1, the directional-hemispherical transmittance of LDPE was most reduced at low angles of incidence. This fraction of radiation decreased with increasing inclination angle. As a consequence, the impact of condensate on the hemispherical-hemispherical transmittance was highest at low inclination angles. When comparing the simulated results with the experimental findings of von Zabeltitz (1987), good agreement can be observed. On a 15 and $30^{\circ}$ inclined PE film, von Zabeltitz (1987) measured a relative solar transmittance reduction of $19 \%$.

\subsection{Clear sky}

3.2.1. Dry state. As was to be expected, it was found that under clear sky conditions, great variations in the transmittance of SG and LDPE occur over time, depending on the orientation and the inclination of the transparent material. The shape of the transmittance curves depended on the glazing orientation and the time. No general conclusions could be drawn on the effect of the inclination angle on the transmittance of the glass plate, since the transmittances of the glass plate and the plastic film increased, decreased or remained constant with increasing inclination angle depending on the simulation circumstances. Similar to the hemispherical-hemispherical transmittance curves under an overcast sky, the hemispherical-hemispherical transmittance curves of SG and LDPE in the dry state were parallel, since the directional-hemispherical transmittance curve of both materials almost coincided. Relatively, the hemispherical-hemispherical transmittance of dry LDPE was 3 to $6 \%$ lower when compared with dry SG.

One specific situation was selected to illustrate the transmittance course as a function of inclination, namely the situation of a S-oriented SG plate and LDPE film during winter (23 December). In that period, condensation frequently occurs due to the relatively low surface temperature of the transparent material, while the highest irradiance levels are received by a S-oriented material. For that day, the average daily hemispherical-hemispherical transmittances of SG and LDPE in the dry and the wet state are illustrated in Fig. 4. The thick lines in the figure represent the relative effect of the condensate on the transmittance.

When comparing Figs 3 and 4, it is clear that the transmittance course as a function of inclination was highly depending on the radiant sky conditions. For the particular case illustrated in Fig. 4-in contrast to the findings under an overcast sky-the lowest transmittance values were found for a horizontal glazing material. For the dry state, the transmittance showed an increasing trend for small inclination angles. For inclination angles higher than $30^{\circ}$, a constant transmittance value of about 81 and $79 \%$ for SG and LDPE, respectively, was found. For a horizontal transparent material, the smallest incidence angle of direct radiation in winter was about $75^{\circ}$ at the mentioned location. This is a result from the fact that direct radiation is highly reflected on the glass plate, which gives rise to the low transmittance value of about $68 \%$ for visible radiation. For inclination angles between 30 and $90^{\circ}$, direct radiation strikes the glass plate at lower incidence angles for which the directional-hemispherical transmittance-and in that way the hemispherical-hemispherical transmittance-is much higher.

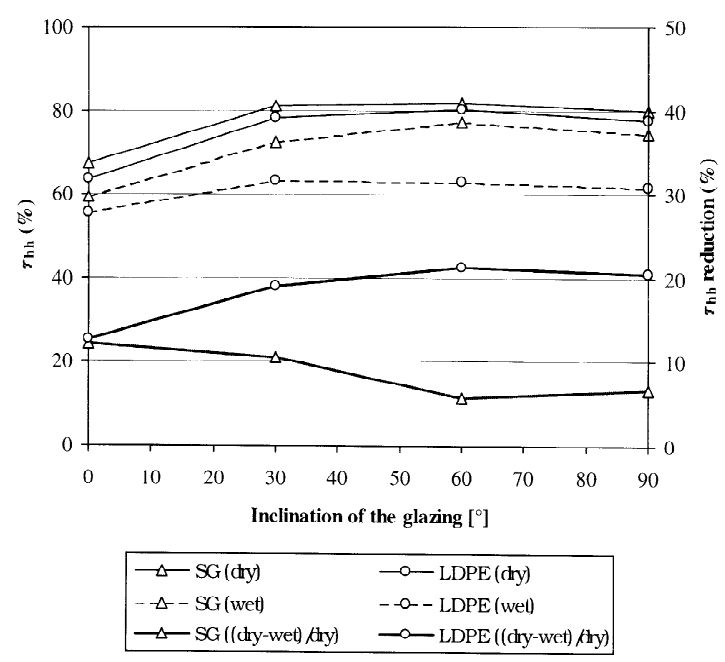

Fig. 4. Average daily hemispherical-hemispherical transmittances of dry and wet south oriented SG and LDPE and the relative transmittance difference between the dry and the wet state under a clear sky on 23 December as a function of the inclination angle of the glazing material. 
Among the situations as specified in Section 2.2, the highest transmittances were found on a S-oriented glass plate $(83 \%)$ during spring and summer. Especially during spring, the transmittance of a S-oriented glass plate was high and quite constant over the range of inclination angles. This can be explained by the high flux of radiation which strikes the transparent material in the range of incidence angles where the directionalhemispherical transmittance is maximal and almost constant $\left(0-40^{\circ}\right)$. For all cases under consideration, the lowest transmittance (36\%) was found on a N-oriented $60^{\circ}$ inclined LDPE film during summer. This low transmittance was due to the high flux of direct radiation striking the transparent material around noon at high angles of incidence.

When comparing the transmittances of $\mathrm{N}$ - and S-oriented inclined transparent materials, the transmittance was found to be always higher for $\mathrm{S}$-oriented materials when compared with $\mathrm{N}$-oriented ones. This was due to the lower mean value of the incidence angle for radiation striking a S-oriented material when compared with a $\mathrm{N}$ oriented one.

3.2.2. Wet state. The impact of the presence of condensate on the transmittance can be deduced from Fig. 4 for the particular situation of a Soriented material during winter. Besides, for all situations under consideration, the average daily transmittance reductions of both materials caused by the condensate are summarised in Fig. 5 .

For each orientation and inclination of both transparent materials and at any moment, Fig. 5 points out that the presence of condensate reduced significantly the transmittance under clear sky conditions. No clear relation between the orientation or the inclination angle on the one hand and the impact of condensate on the transmittance on the other hand was found. The relative effect of condensate on the transmittance of both materials varied between 6 and 21\%. Both extreme values occurred on a S-oriented material. However, the minimum value of $6 \%$ corresponded with glass and the maximum value with plastic.

The effect of condensate on the reduction of the hemispherical transmittance of SG and LDPE was clearly different, due to the differences in the angular transmittance curves of both materials. The highest differences between both materials were found for a S-oriented 30 or $60^{\circ}$ inclined material. For that orientation and inclination, the incidence angles of high intensity radiation around noon were small. For this range of inci-
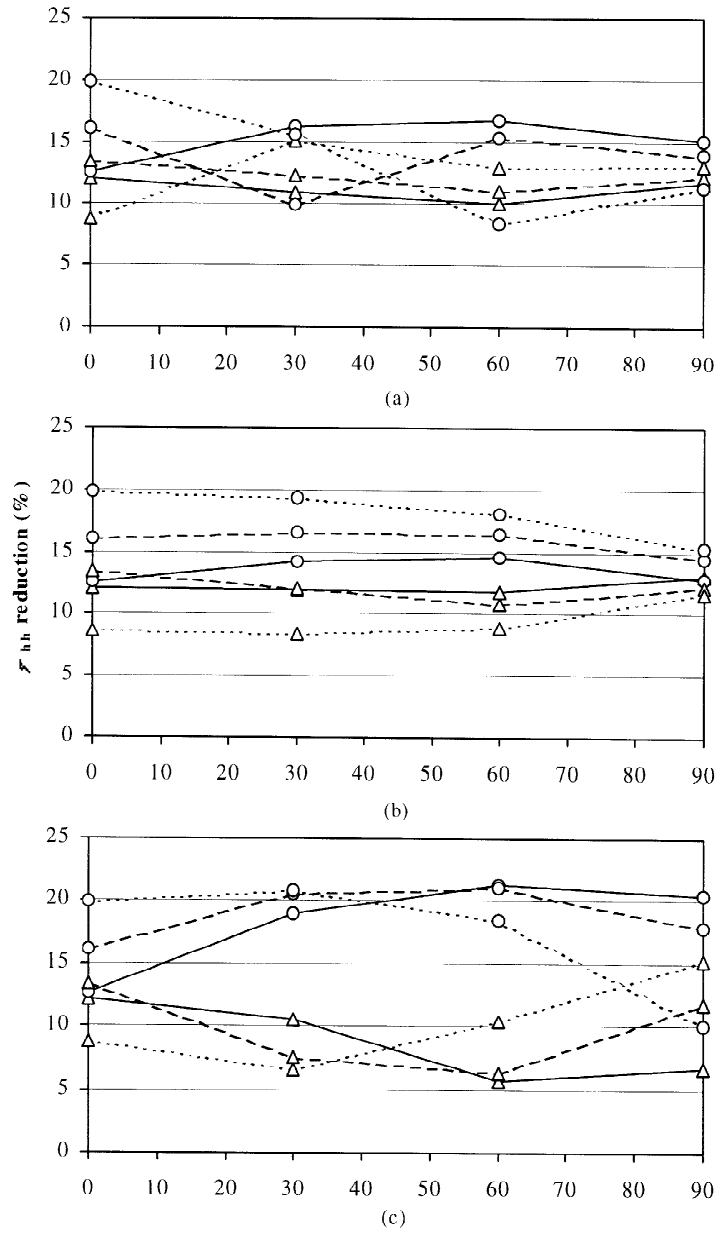

Inclination of the glazing $\left[{ }^{\circ}\right]$

\begin{tabular}{|ll|}
\hline$\Delta-$ SG (Winter) & $-0-$ LDPE (Winter) \\
$-\Delta-$ SG (Spring) & $-0-$ LDPE (Spring) \\
$\cdots \Delta \cdots$ SG (Summer) & $\cdots-.$. LDPE (Summer) \\
\hline
\end{tabular}

Fig. 5. Relative reduction of the hemispherical-hemispherical transmittances of (a) north, (b) east, and (c) south oriented SG and LDPE due to the presence of condensate on 23 December (winter), 22 March (spring), and 22 June (summer) as a function of the inclination angle of the glazing material under a clear sky.

dence angles, the differences between the angular transmittances of wet SG and wet LDPE were maximal.

For some orientations and at some moments, the influence of condensate was almost independent of the inclination angle of the transparent material (for instance N-oriented glass during winter and spring). For other cases, a great variation was observed (for instance S-oriented glass during spring and summer), which means that the average incidence angle of the total incident radiation obviously differed in time.

The calculations revealed that condensate exerted the greatest effect on glass when relatively great fractions of radiation struck the transparent 
material at incidence angles in the range of 50$75^{\circ}$. At these incidence angles, the directionalhemispherical transmittance is most affected by the condensate as was shown in Fig. 1. These conditions occur for instance in summer on a vertical S-oriented glass plate (see Fig. 5c). On the contrary, when direct radiation struck the transparent material at small incidence angles, condensate had a small influence on the transmittance of glass. The transmittances of 30 and $60^{\circ}$ inclined glass plates were most affected by condensate when exposed towards the north. The effect of condensate on the transmittance of a vertical Eoriented SG plate was almost independent of the time of the year.

In most cases, clear differences were found between the effect of condensate on the transmittance during the winter when compared with the summer. During winter, when the radiation flux is lowest and the chance that condensation occurs is highest, the relative transmittance reduction due to condensate on SG varied between 6 and 13\%. During summer, values ranging from 7 to $15 \%$ were found.

The relative effect of the condensate on the transmittance of LDPE varied between 8 and $21 \%$. It was shown in Fig. 1, that the angular transmittances of LDPE in the dry and the wet state differ most for small angles of incidence up to $30^{\circ}$. These conditions occur especially in winter on a S-oriented $60^{\circ}$ inclined surface, for which the highest relative impact of condensate on the transmittance is found. The lowest effect of condensate occurred when radiation struck the transparent material at high angles of incidence, for which the angular transmittance of LDPE is least affected. These circumstances occur for instance in summer on a $\mathrm{N}$-oriented $60^{\circ}$ inclined surface. Actually, it can be stated, as clearly observed in Fig. 4, that the effect of condensate on the transmittance was proportional to the transmittance of dry LDPE. This relation was not valid for SG.

In general, the relative effect of the condensate on the average daily transmittance was obviously higher for LDPE when compared with SG. At a particular moment or inclination of the glazing material (for instance a S-oriented vertical surface during summer), however, a higher relative effect of the condensate on the transmittance of SG can be found when compared with LDPE. Under these latter circumstances, a great fraction of radiation strikes the transparent materials at angles between 50 and $75^{\circ}$.

Of course, it should be kept in mind that the presence of condensate on the inside surface of the transparent material is time dependent. Condensation can be found on any day of the year. However, condensate is especially formed during periods of low insolation, namely during winter, but also in summer, near sunset and sunrise, and during cloudy periods.

\section{CONCLUSIONS}

Hemispherical-hemispherical transmittance values of single glass and low-density polyethylene in the visible radiation range were calculated using experimentally determined angular transmittances. Particularly, the impact of the presence of condensate on the hemisphericalhemispherical transmittance of both glazings was determined under an overcast and a clear sky for several orientations and inclinations of the glazing. For both materials, condensate always reduced the total transmittance under outside radiant conditions. Quantitatively, however, the impact of the condensate differed remarkably due to the differences in the condensate patterns formed on both glazings. The relative effect of condensate on the transmittance varied between 6 and $15 \%$ for SG and between 8 and $21 \%$ for LDPE. The orientation and the inclination angle of the glazing showed no clear relation with the impact of condensate on the transmittance. In most cases, condensate reduced the transmittance of LDPE the most when compared with SG. In the case of LDPE, it was found that the effect of condensate on the transmittance was proportional to the transmittance of dry LDPE. This was not found for SG. The results revealed that under outside radiant conditions, the presence of condensate could considerably reduce the transmittance of, and as a consequence the irradiance level, inside a solar energy system. Therefore, the impact of the presence of condensate on the transmittance should be included in the design and performance calculations of solar energy systems if condensation is expected.

\section{NOMENCLATURE}

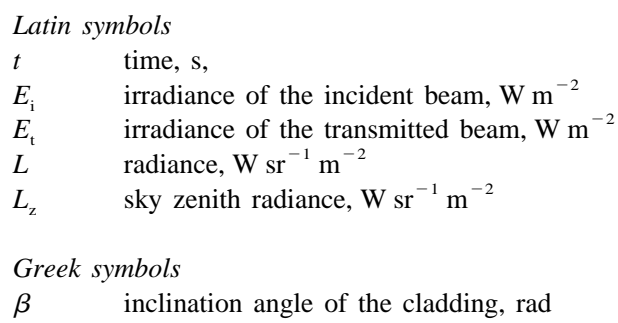


$\phi \quad$ azimuthal angle of a sky point, rad

$\phi_{\text {sun }} \quad$ azimuthal angle of the sun, rad

$\theta \quad$ altitude angle of a sky point, rad

$\theta_{\mathrm{i}} \quad$ incidence angle of a sky point, $\mathrm{rad}$

$\theta_{\text {sun }} \quad$ altitude angle of the sun, rad

$\tau_{\mathrm{dh}} \quad$ directional-hemispherical transmittance, -

$\tau_{\text {hh }} \quad$ hemispherical-hemispherical transmittance, transmittance, -

$\xi \quad$ angle between the sun and a sky point, rad

$\zeta \quad$ zenith angle of a sky point, rad

\section{Abbreviations}

A cladding point

E east

LDPE low-density polyethylene n: normal

$\mathrm{N}$ north

$\mathrm{P} \quad$ sky point

S south

SG single glass

W west

Z zenith $\bar{\tau}_{\text {hh }} \quad$ weighted average hemispherical-hemispherical

Hutchins M. G. and Ageorges P. (1993) Angular dependent spectral optical properties of architectural glazings: results of an inter-laboratory comparison of measurements. In Optical Materials Technology for Energy Efficiency and Solar Energy Conversion XII, Lampert C. M. (Ed.), Vol. 2017, pp. 13-24. Proceedings of the SPIE.

Karlsson J. and Roos A. (2000a) Angle-resolved optical characterization of an electrochromic device. Solar Energy 68(6), 493-497.

Karlsson J. and Roos A. (2000b) Modelling the angular behaviour of the total solar energy transmittance of windows. Solar Energy 69(4), 321-329.

Karlsson J., Rubin M. and Roos A. (2001) Evaluation of predictive models for the angle-dependent total solar energy transmittance of glazing materials. Solar Energy 71(1), 23-31.

Kittler R. (1967) In Proceedings of the CIE Intercessional conference: sunlight in buildings, Newcastle-Upon-Tyne.

Mbise G. W., Le Bellac D., Niklasson G. A. and Granqvist C. G. (1997) Review article: Angular selective window coatings: theory and experiments. J. Phys. D: Appl. Phys. 30, 2103-2122.

Monteith J. L. (1973). Principles of Environmental Physics, Arnold, London.

Monteith J. L. and Unsworth M. H. (1990). Principles of Environmental Physics, Chapman and Hall, New York.

Palmer S., Mbise G. W., Niklasson G. A. and Granqvist C. G. (1996) Angular selective optical properties of thin films: measurement of polar and azimuthal transmittance. Solar Energy Mater. Solar Cells 44, 397-403.

\section{REFERENCES}

Aggarwal S. and Narayan A. (2000) Computer based thermal modelling of double condensing chamber solar still. In Renewable Energy, Sayigh A. (Ed.), pp. 1114-1117, Pergamon, Amsterdam.

Breuer J. J. G. (1981) Energy measurements on various greenhouse coverings. IMAG 81-1, Wageningen.

Briscoe B. J. and Galvin K. P. (1991) The effect of surface fog on the transmittance of light. Solar Energy 46(4), 191-197.

Edwards R. I. and Lake J. V. (1964) Transmission of solar radiation in a large-span east-west glasshouse. J. Agric. Engng. Res. 9(3), 245-249.

Edwards R. I. and lake J. V. (1965) Transmission of solar radiation in a large-span east-west glasshouse. II. J. Agric. Engng. Res. 10(2), 125-131.

El-Bahi A. and Inan D. (1999) A solar still with minimum inclination and coupled to an outside condenser. In Proceedings of ISES Solar World Congress, Jerusalem, Israel, pp. 1277-1282.

Fechner H. and Bucek O. (1999) Solar air collectors-investigations on several series-produced collectors. In Proceedings of ISES Solar World Congress, Jerusalem, Israel, pp. 1103-1108.

Geoola F., Kashti Y. and Peiper U. M. (1998) A model greenhouse for testing the role of condensation, dust and dirt on the solar radiation transmissivity of greenhouse cladding materials. J. Agric. Engng. Res. 71(4), 339-346.

Geoola F., Peiper U. M. and Geoola F. (1994) Outdoor testing of the condensation characteristics of plastic film covering materials using a model greenhouse. J. Agric. Engng. Res. 57(3), 167-172.

Gombert A., Steinhart J., Sanowski C., Forcht K., Brucker F., Graf W. and Koehl M. (1995) Measurement of the hemispherical reflectance and transmittance at variable angles of incidence. In Optical Materials Technology for Energy Efficiency and Solar Energy Conversion XIV, Lampert C. M., Deb S. K. and Granqvist C. G. (Eds), Vol. 2531, pp. 308-316. Proceedings of the SPIE.

Hsieh C. K. and Rajvanshi A. K. (1977) The effect of dropwise condensation on glass solar properties. Solar Energy 19, 389-393.
Pieters J. G. and Deltour J. M. (1998) Effect of latent heat phenomena on thermal insulation and inside climate of greenhouses. J. Therm. Env. Build. Sc. 22, 9-31.

Pieters J. G., Deltour J. and Debruyckere M. (1997) Light transmission through condensation on glass and polyethylene. Agric. For. Meteorol. 85, 51-62.

Platzer W. J. (1992) Directional-hemispherical solar transmittance data for plastic honeycomb-type structures. Solar Energy 49(5), 359-369.

Pollet I. V. and Pieters J. G. (1999) Condensation and radiation transmittance of greenhouse cladding materials. Part I: laboratory measuring unit and performance. J. Agric. Engng. Res. 74, 369-377.

Pollet I. V. and Pieters J. G. (2002a) Effect of inclination angle on the light transmittance of condensate covered homogeneous thin transparent materials. Solar Energy Mater. Solar Cells 73, 435-439.

Pollet I. V. and Pieters J. G. (2002b) visible radiation transmittances of dry and condensate covered glass and plastic greenhouse cladding. Agric. For. Meteorol. 110(4), 285298.

Rea M. S. (1993). Lightening Handbook. Reference and Application, 8th Edition, Illuminating Engineering Society of North America, New York.

Roos A. (1997) Optical characterization of coated glazings at oblique angles of incidence: measurements versus model calculations. J. Non-Cryst. Solids 218, 247-255.

Rubin M., Powles R. and Von Rottkay K. (1999) Models for the angle-dependent optical properties of coated glazing materials. Solar Energy 66(4), 267-276.

Rubin M., Von Rottkay K. and Powles R. (1998) Window optics. Solar Energy 62(3), 149-161.

Schultz W. and Bartnig K. H. (1996) Evaluation of the nondrip properties of greenhouse cladding films. Plasticulture 111, 23-35.

Van Nijnatten P. A. and Spee C. I. M. A. (1997) Determination of directional optical properties of an electrochromic device. J. Non-Cryst. Solids 218, 302-306.

von Zabeltitz C. (1987) Folien als Gewachshausbedachung (in German). Landtechnik 42, 326-327. 